\title{
Surgery and Gerontology
}

\author{
Siniša Franjić* \\ Independent Researcher \\ *Corresponding author: Siniša Franjić, Independent Researcher, E-mail: sinisa.franjic@gmail.com
}

Received: December 18, 2021; Accepted: December 25, 2021; Published: December 31, 2021

\begin{abstract}
Older people represent a very specific and vulnerable age group whose share is progressively increasing, which significantly affects the health, social, educational and economic structure of the entire population of any country in the world. Gerontology is a scientific discipline that studies aging in the broadest sense, ie its clinical, biological, economic, social and psychological aspects. This results in a comprehensive, holistic approach to health care for the elderly, which ensures the improvement of all forms of health care for the elderly. One of the branches of medicine that considers the protection of the health of the elderly is surgery.
\end{abstract}

Keywords: Surgery, Elderly, Comorbidities, Care

\section{Introduction}

In recent years, there has been a growing recognition of the role for geriatric medicine specialists in the care of older surgical patients [1]. This has been fueled in part by the increasing numbers of older people undergoing elective and emergency surgery and in part by the increasing medical complexity of older surgical patients. The increase in numbers is due to changing global demographics, resulting in an age-related increase in the prevalence of degenerative and neoplastic pathology, for which surgery is often the best treatment option, and to advances in surgical and anesthetic technique. Furthermore, patient expectations and health care professional attitudes and behaviors have evolved, with impetus provided by legislation against age discrimination. The overall impact is that rates of surgical procedures in older adults are now significantly higher than in any other age group.

Although rates of surgery in the older population have increased, they have not kept pace with the observed prevalence of conditions requiring surgery. It appears that surgery may still not be offered to older patients where it would be offered to younger patients, either for symptomatic or curative benefit. For example, the rates of hip arthroplasty decline steadily beyond the age of 70 years, as do resection rates for curable cancer across a range of tumor sites. This is despite the fact that older adults have much to gain from surgery for symptomatic control (as in joint replacement surgery) and improved survival (as in colorectal cancer). The apparently limited access to surgery seen in some older adults may occur for a number of different reasons, but a likely contributor is the complex analysis of risk or harm versus benefit of surgery in older adults. It requires an understanding of not only the surgical and anesthetic issues, but also of life expectancy with and without surgery, alternative treatment options, modifiable risk factors, and management of predictable and unpredictable postoperative complications. Such analysis needs to be presented in a manner appropriate to the patient to facilitate shared decision making.
The complexity of the older surgical population, which makes the assessment of the risk-to-benefit ratio difficult, relates to the association of aging with physiologic decline, multimorbidity, and frailty, all of which are independent predictors of adverse postoperative outcome. With such a profile, it is no surprise that in comparison to the younger population, older patients suffer from higher rates of postoperative morbidity and mortality when undergoing emergency and elective surgery across various surgical subspecialties. Furthermore, in older adults, a surgical procedure with associated hospitalization is more likely to result in impaired functional recovery, with a consequent need for rehabilitation, complicated hospital discharge, and increased home care or new institutionalization. This complexity in older surgical patients presents challenges throughout the surgical pathway, from the preoperative decision making phase to medical management in the postoperative period.

Geriatric citizens in the United States are the most rapidly growing segment due to the aging baby boomer generation [2]. This generation will live longer than the preceding and will have access to improved health care. Because these physically active elderly will remain living independently and longer, traumatic injuries can be expected to increase. In addition, there are numerous physiologic alterations that occur with aging, and special consideration should be given to the elderly patient from a medical and surgical standpoint. Multiple comorbidities may also be present in this population lending to higher complications, longer hospital stays, and a higher case fatality rate. Moreover, disposition barriers often exist and include the need for short- and long-term rehabilitation. Finally, traumatic injuries have the ability to change the patient's independent living status and increase the need for admission to skilled nursing facilities. Complex end-of-life decisions and discussions are often also required in this population. Trauma and acute care surgeons should be knowledgeable about the specifi $\mathrm{c}$ needs of the geriatric critically ill patient. 


\section{Comorbidities}

The presence of coexisting disease-in particular anemia, diabetes, and cardiac, respiratory, and renal disease-increases the risk of adverse postoperative outcome [1]. Although each individual condition increases this risk, a combination of more than three coexisting conditions (multimorbidity) is highly predictive of postoperative complications, poor functional outcome, and mortality. Because increasing age is associated with multimorbidity, with more than $40 \%$ of community-dwelling people older than 70 years living with multimorbidity, older adults presenting for surgery are a vulnerable population. Various scores are available to describe and measure comorbidities (e.g., the Charlson Comorbidity Index). These are useful for comparison between patient groups and stratification of risk and thus for coding and research, but their clinical utility in the surgical population is limited.

Furthermore, the severity of the coexisting condition and its related complications is more important in affecting outcome than merely its presence. For example, poorly controlled diabetes associated with untreated diastolic heart failure is of more significance than well-controlled diabetes and mild optimized chronic obstructive pulmonary disease (COPD), despite the fact that the comorbidity count would be the same. Recognition of the impact of comorbidity on postoperative outcome has led to the publication of resources to guide perioperative assessment and optimization of specific comorbidities. These resources include guidelines covering cardiac disease (e.g., coronary artery disease, valve disease, cardiac failure), anemia, and diabetes. Interestingly, although it is intuitive that optimization of such comorbidities should reduce the risk of poor outcome, there are little data to support such hypotheses (e.g., there are no reliable studies to date demonstrating that preoperatively reducing hemoglobin A1c [HbAlc] levels in patients with diabetes results in improved postoperative outcomes).

\section{Risks}

Patients with angina, recent myocardial infarction (MI), arrhythmias, congestive heart failure (CHF), and diabetes are at significantly increased risk for perioperative MI, heart failure, or arrhythmias [3]. An increased risk for cardiac complications is also present in elderly patients and those with abnormal electrocardiograms (ECGs), low functional capacity, history of stroke, and uncontrolled hypertension.

Surgeries may be classified as high-, intermediate-, or low-risk procedures. Those posing a high risk for cardiac complications (greater than $5 \%$ cardiac risk) include vascular surgeries, emergency surgeries, and surgeries associated with increased blood loss or large fluid shifts. Intermediate-risk surgeries ( $1 \%$ to $5 \%$ cardiac risk) include most intrathoracic, intraperitoneal, and orthopaedic procedures. Low-risk procedures (less than $1 \%$ cardiac risk) include cosmetic procedures, cataract operations, and endoscopies.

Patients at risk for pulmonary complications include those with lung disease-for example, asthma or chronic obstructive pulmonary disease (COPD) - obesity, a history of smoking, and undiagnosed cough or dyspnea. Procedures that increase the risk for pulmonary complications are primarily abdominal or thoracic surgeries, with the rule being that the closer the surgery is to the diaphragm, the higher the risk of complications.

Wound infections are the most common infectious complications following surgery, followed by pneumonia, urinary tract infections, and systemic sepsis. Diabetes and vascular disease are patient factors associated with an increased risk for wound infections. Surgeries with potential spillage of infectious material, such as abscess drainage or gastrointestinal surgery, pose a higher risk of postoperative infections. Instrumentation of the urinary tract, as occurs during bladder catheterization or genitourinary surgery, can lead to the development of urinary tract infections.

\section{Complications}

Caring for the older surgical patient presents unique problems: older individuals present with more advanced disease, have more comorbidities and suffer more complications than younger patients [4]. Appropriate patient selection and perioperative care is essential for optimizing surgical outcomes in this population. The benefits of the most commonly performed surgical procedures are well established. Colon resections increase colorectal cancer-free survival, and hip replacements significantly improve joint pain and functional ability. These benefits, however, must be weighed against the risk of mortality, morbidity, and decreased quality of life that sometimes follow these operations.

Nationally representative large cohort studies provide the most realistic information about surgical risk in older adults. In a national sample of patients undergoing highrisk cancer operations, patients older than age 80 years who were undergoing esophageal resections had an operative mortality of $20 \%$ with only $19 \%$ of patients experiencing long-term survival beyond 5 years. Morbidity after surgery in older adults is also high. Surgical complications, such as wound infections, bleeding, and need for reoperation, are not more frequent, but the occurrence of nonfatal postoperative complications is independently associated with decreased long-term survival.

Major operations may also result in a diminished quality of life by causing postoperative cognitive and functional decline. The risk of postoperative cognitive dysfunction following cardiac surgery is well studied, and there is now increasing evidence that postoperative cognitive dysfunction also occurs after noncardiac procedures. Up to $10 \%$ of patients older than age 60 years suffer from memory problems 3 months out from noncardiac surgery. It is unclear whether it is acute illness, anesthesia, or surgery that is the primary contributor to this condition. Functional changes following surgery can also be prolonged and irreversible. More than half of patients undergoing abdominal operations experience significant functional decline that persists for up to a year after surgery. A recent study assessing functional status following colectomy in nursing home residents found that the most active patients suffer the greatest decline as they have the most to lose. These findings emphasize the importance of addressing the risk of functional decline in all older patients, even the most active. For some patients, loss of independence weighs heavier than mortality when deciding whether to undergo a high-risk operation. Awareness of 
these risks is essential for appropriate patient selection. It also allows clinicians to offer a realistic expectation of outcomes, which, in turn, informs decision making by the older individual and their families.

\section{Diabetes}

During their lifetime, most patients with diabetes will require some form of surgery, and the likelihood increases as age advances [5]. Nowadays, a considerable amount of major surgery is undertaken in the elderly (e.g. coronary artery bypass grafts, peripheral vascular and aneurysm surgery, removal of malignancies), of whom more are proportionately likely to have diabetes than at the earlier stages of their lives. Even during the past few years in England, there has been a $16 \%$ increase in coronary artery bypass grafts and a similar increase in hip replacements in the elderly. Surgical practice is also changing in many countries, with an increasing number of day-case procedures and shorter postoperative hospital stays. Diabetes management in the elderly is also changing with the increasing use of insulin, and sometimes with more complicated multiple injection regimens and even occasionally the use of insulin pumps.

Although carefully planned and executed surgery is highly successful in the elderly, such patients with diabetes may tolerate metabolic and infective complications less well than younger subjects. Diabetes per se should never be a reason to decide not to operate on an elderly patient, but it is a reason for careful planning and management - whether preoperatively, perioperatively or postoperatively.

\section{Tumors}

Surgery is the most important modality of treatment for many of the common tumors in the elderly [6]. For instance, surgical resection is required for cure of early-stage colon cancer but is also frequently pursued for patients with metastatic disease, to prevent the likely complications of obstruction and bleeding. Studies in the surgical oncology literature demonstrate that advanced age does not preclude surgery; however, patients in these studies are usually selected carefully. Mortality from elective surgery increases only minimally, if at all, with advancing age.

However, if an older person requires emergency surgery, the operative risk can be at least twice as high. Mortality rates for elective colon cancer resection range from $4 \%$ to $21 \%$ but rise to over $50 \%$ if the procedure is for an emergency. This illustrates the geriatric principle of diminished functional reserve, in which aging is associated with a diminution of the functional capacity of multiple organ systems and the impact of a profound stress to the system is magnified compared to a younger person.

Nonetheless, surgery should be considered an important part of palliative treatment, even for older patients with poor prognosis. For example, patients with pancreatic cancer, of whom two-thirds are older than 65 , have a 5 -year survival of about $5 \%$. Approximately $50 \%$ of these patients will require surgery for biliary or gastric obstruction, which are the common complications of this disease.

Performance status falls immediately after surgery for all patients, young and old. This status improves for younger patients, but older patients may not return to their functional baseline. Hence, surgical reports that describe short-term postoperative morbidity and mortality rates may be missing the outcomes that are most important for geriatric cancer patients: increasing dependence in activities of daily living, which leads to loss of autonomy. Some centers are exploring newer techniques, such as the role of laparoscopic surgery for colon resection in the elderly. This may lead to less postoperative pain, diminution of postoperative ileus, and a shorter hospital stay.

\section{Health Care}

As our population is aging, older patients are living longer with chronic illness [7]. Discussion on the goals of care should be initiated with the admission of geriatric patients. A multidisciplinary approach involving the patient and family with the discussion on the risk and benefits will allow the patients to make informed decision toward the end of life. Advance care planning can decrease the suffering, increase the quality of life, and improve the experience of family members and decrease healthcare costs. Establishing goals of care that correspond with the patient's values and preferences; and communication between the patient and all those involved in their care should be part of the assessment of any geriatric patient for emergency general surgery. It is important for surgeons to identify high-risk patients and initiate the discussion of a definitive curative surgery vs. a temporizing procedure based on the goals of care.

Managing risks and predicting postoperative outcomes in elderly patients who undergo emergency general surgery is a complex process due to their acute presentation, which renders many preoperative preparations difficult to apply. However, there are certain preoperative and most often postoperative opportunities to improve outcomes. Therefore, focusing on preoperative and postoperative outcomes in such patients should be the target for both the surgeon and the hospital. In comparison to age alone, frailty is used as an objective tool to predict the postoperative outcomes in elderly and helps surgeons to formulate their decisions in managing this group of patients. Geriatric consultation is recommended in the hospital setting as it is associated with reduction in mortality rates, hospital length of stay, as well as lower costs of care.

The context of geriatric care encompasses multiple levels, stretching from primary care, through acute hospitalization, acute and subacute rehabilitation, nursing home care, and hopefully back to sufficient function to require additional primary care [8]. By the nature of their practices, anesthesiologists and geriatricians have different approaches to patient care and the time frame over which such care occurs. In communicating with patients and geriatricians, one should understand that expectations for recovery are frequently different than in younger patients, marked by issues of maintenance of function and independence. There is an evolving understanding that specific approaches taken in the perioperative period have an impact that remains apparent months to years following surgery. Integrating care across this continuum can be diffi cult but invariably improves patient outcomes.

\section{Palliative Care}

Many of the patients who come into contact with the community palliative care clinical nurse specialist will also have undergone 
surgery of some description, as part of their cancer treatment [9]. Radical surgery is probably the most effective treatment in cancer management. However, palliative surgery also plays a part in symptom relief; for example, internal fixation of a pathological fracture or to relieve an oesophageal or bowel obstruction. These procedures will have no impact on the course of the disease itself, but may bring about considerable symptom relief, therefore improving quality of life. As identified previously, patients require information about the surgery, what the operation will involve and the projected time scale for recovery. Many of the surgical procedures may be fairly minor in terms of surgical time involved, but risks versus benefits must be considered. Patients are understandably anxious about surgery and need reassurance and an opportunity to express their fears and concerns. In addition to providing information, the community palliative care clinical nurse specialist can also respond to the concerns of the patient, correcting misconceptions, and assist the patient to discuss his or her worries.

After treatment, whether it is radiotherapy, chemotherapy or surgery, etc., the patient will require ongoing support for weeks, months or in some cases years. The follow-up will usually be with the oncologist, but increasingly the general practitioner and the primary health care team are being relied upon to monitor the patient and refer back to the oncologist if required. It is likely that in the future the community palliative care clinical nurse specialist will play an increasing role in managing the follow-up of patients, in tandem with the general practitioner. This may be reassuring for patients, but visits to the doctor's surgery or home visits by the primary health care team and even the community palliative care clinical nurse specialist may be seen as a constant reminder of the illness and an intrusion into the patient's daily life. Patients may also become very anxious prior to medical/nursing appointments or visits, whether at the hospital, surgery or in their own homes, as discussions may reveal new symptoms that suggest progression of their disease. After treatment, fear of the cancer returning or progressing means that it is difficult for patients to return to 'normal', and contact with their health care team may be frequent as the patient looks for reassurance and support. It is important for the professionals to achieve a balance, whereby patients feel well supported and know where to get help, but also are allowed to continue living with their cancer or other life-threatening illness and enjoy some semblance of 'normality' in their lives.

When treatment finishes, whether curative or palliative, patients may feel that their 'security' has ended. They may have a sense that nothing is actually happening at present to stop their cancer. This can be a difficult time for patients and they need support and reassurance that their symptoms and cancer are being monitored. Some patients may find it difficult to live with the uncertainty that comes with their disease and need the opportunity to discuss their concerns and fears. The community palliative care clinical nurse specialist can negotiate with patients appropriate contacts, whether by telephone or visiting, to support them with their ongoing complex emotional needs during this difficult time. The threat of physical deterioration is ever present and patients who experience a recurrence of their cancer report that the news can cause greater shock and devastation than the original diagnosis.

\section{Procedure}

All patients should be aware that there are risks attached to all forms of surgical intervention [10]. Usually, the risks are low and worth taking. Unfortunately, the risks rise with increasing age, but the benefits can still be enormous. Much skill and experience is needed from all those involved in the surgical care of elderly patients-their nurses, anaesthetists, surgeons and therapists.

The national confidential enquiry into peri-operative deaths 'at the extremes of age' has highlighted many problems relating to the increased mortality of very elderly patients within surgical departments. The report recommends that emergencies in old age should be dealt with promptly (within 24 hours), with the most experienced staff undertaking the work. It is often a very fine balance as to how much time can be devoted to improving the general condition of a very sick, elderly patient before embarking on an operation.

It should also be appreciated that, just because a procedure is possible, it is not always advisable or desirable. Mentally competent patients will be able to make this difficult decision for themselves once the situation has been explained to them. Some will have indicated in advance (by a living will or an advance directive) their wishes in these matters. In many other cases, the difficult choices will have to be made by others in the best interests of the patient. In these situations, experienced practitioners, assisted by the patient's family and friends, are most likely to make the correct decision.

\section{Conclusion}

Most biological functions of man reach their peak before the age of 30 , after which they decline linearly; this reduction can be critical in a state of stress, but it affects daily activities almost in no way. Therefore, diseases, not normal aging, are the main reasons for loss of function in old age. Often this decline with age is at least partly due to lifestyle, behavior, diet or environment, which can be influenced. Thus exercise can prevent or improve maximal load tolerance, muscle strength, and glucose tolerance in healthy but sedentary older individuals. The effects of uncontrollable aging are smaller than previously thought, allowing many to have a healthier and stronger age.

\section{Reference}

1. Dhesi JK, Partridge J, Fillit HM, Rockwood K, Young J (2017) Surgery and Anesthesia in the Frail Older Patient. Brocklehurst's Textbook of Geriatric Medicine and Gerontology, Eighth Edition, Elsevier, Philadelphia, USA, pp: 232-233.

2. Gordy S, Trunkey D, Yelon JA, Luchette FA (eds). (2014) Changing Demographics of the American Population Geriatric Trauma and Critical Care, Springer Science, Business Media, New York, USA, pp: 3. [crossref]

3. Lipsky MS, King MS (2011) Blueprints Family Medicine Third Edition, Wolters Kluwer, Philadelphia, USA, pp: 19.

4. Oresanya L, Finlayson E, Williams BA, Chang A, Conant R, et al. (2014) Perioperative Care in Older Surgical Patients . Current Diagnosis and Treatment-Geriatrics, Second Edition, McGraw-Hill Education, New York, USA, pp:95.[crossref]

5. Gill G, Benbow S, Sinclair AJ (ed) (2009) Managing Surgery in the Elderly Diabetic Patient Diabetes in Old Age, Third Edition, Wiley-Blackwell, John Wiley \& Sons, Chichester, UK, pp: 349.

6. Sacks NR, Abrahm JL, Morrison RS, Meier DE (eds) (2003) Cancer Geriatric Palliative Care, Oxford University Press, Inc., Oxford, UK, pp:130. 
7. Joseph B, Hamidi M, Brown CVR, Inaba K, Martin MJ, et al. (2019) Emergency General Surgery in the Elderly, Emergency General Surgery-A Practical Approach, Springer International Publishing AG, Cham, Switzerland, pp: 459-460. [crossref]

8. Silverstein JH, Rooke GA, Reves JG, McLeskey CH (eds). (2008) The Practice of Geriatric Anesthesia Geriatric Anesthesiology, Second Edition, Springer Science, Business Media, LLC, New York, USA, pp: 3.
9. Aitken AM, Chichester UK (2009) Community Palliative Care-The Role of the Clinical Nurse Specialist, Wiley-Blackwell, John Wiley \& Sons Ltd, pp: 54-56.

10. Rai GS, Webster S (2000) Elderly Care Medicine, Cavendish Publishing Limited, London, UK, pp: 95.

\section{Citation:}

Franjić S (2021) Surgery and Gerontology. Sur Res Rep Volume 4(1): 1-5. 